\title{
Análise da Ocorrência de Coleópteros em Plantios de Eucalyptus camaldulensis Dehn. em Cuiabá, MT
}

\author{
José Renato Maurício da Rocha ${ }^{1}$, Alberto Dorval², Otávio Peres Filho², \\ Marcelo Dias de Souza ${ }^{1}$, Reginaldo Brito da Costa ${ }^{2}$
}

${ }^{1}$ Programa de Pós-Graduação em Ciências Florestais e Ambientais, Faculdade de Engenharia Florestas - FENF, Universidade Federal de Mato Grosso - UFMT

${ }^{2}$ Departamento de Engenharia Florestal, Faculdade de Engenharia Florestas - FENF,

Universidade Federal de Mato Grosso - UFMT

\begin{abstract}
RESUMO
Este trabalho teve por objetivo analisar a ocorrência das subfamílias Bostrichinae, Platypodinae e Scolytinae em reflorestamento de Eucalyptus camaldulensis, no município de Cuiabá, Estado de Mato Grosso, nos períodos de estiagem e de chuva. As coletas foram realizadas quinzenalmente entre os meses de abril de 2008 e março de 2009, com o uso de seis armadilhas etanólicas. Foram realizados estudos qualitativos e quantitativos, entre os quais: flutuação populacional, frequência, constância, abundância, dominância e diversidade. Constatou-se a ocorrência de 26 espécies, sendo a subfamília Scolytinae a que obteve maior número de espécies e de indivíduos, em ambos os períodos. As espécies Hypothenemus eruditus e Xyleborus affinis, nos meses de estiagem, e Xyleborus ferrugineus, no período de chuva, foram quantitativamente as mais representativas.
\end{abstract}

Palavras-chave: coleópteros, coleobrocas, diversidade, eucalipto.

\section{Analysis of the Occurrence of Beetles in Plantations of Eucalyptus camaldulensis Dehn. in Cuiaba, MT}

\begin{abstract}
The purpose of this work was to analyze the occurrence of the Bostrichinae, Platypodinae and Scolytinae subfamilies in the planting of Eucalyptus camaldulensis in Cuiaba, State of Mato Grosso, in the dry and rain seasons. The collections were performed fortnightly between April 2008 and March 2009, with the use of six ethanolic traps. Qualitative and quantitative studies were carried out concerning population fluctuation, frequency, constancy, abundance, dominance and diversity. Twenty-six species were found, with the Scolytinae subfamily yielding the largest amount of species and individuals in both periods. Hypothenemus eruditus and Xyleborus affinis in the dry season and Xyleborus ferrugineus in the rain season were the most quantitatively representative species.
\end{abstract}

Keywords: Coleoptera, wood borers, diversity, eucalypt. 


\section{INTRODUÇÃO}

No Brasil, espécies do gênero Eucalyptus são plantadas em quase todas as regiões, em razão da sua capacidade de se adaptar aos mais diferentes tipos de habitats, além de seu crescimento ser mais rápido em relação a outras espécies florestais. Segundo Del Quiqui et al. (2001), o Eucalyptus camaldulensis é uma das espécies mais importantes dentro do gênero Eucalyptus, por ser a mais adequada a zonas críticas, nas quais as deficiências hídricas e os problemas relacionados ao solo são fatores limitantes para outras espécies.

De acordo com Moraes et al. (1997), essa capacidade de adaptação às condições ecológicas muito variadas, aliada ao grande número de procedências existentes, à rapidez de crescimento e à vigorosa brotação de cepa, confere ao E. camaldulensis destaque como espécie de superior plasticidade em termos de adaptação às condições brasileiras, em comparação com outras do mesmo gênero. No entanto, de acordo com Oliveira et al. (2001), monoculturas como as de eucalipto podem favorecer a ocorrência de pragas, gerando a necessidade de se desenvolverem técnicas apropriadas, para reduzir eventuais danos causados pelas mesmas.

Em geral, plantios homogêneos de Eucalyptus spp. são ambientes susceptíveis para a ocorrência de surtos populacionais de espécies de insetos-pragas. Dentre estes, as coleobrocas das subfamílias Bostrichinae, Platypodinae e Scolytinae são consideradas com potencial para promoverem danos em reflorestamento, inclusive em nível econômico. Algumas espécies das subfamílias Scolytinae e Platypodinae são conhecidas como "besouros-de-ambrosia", pois abrem galerias profundas, atingindo o alburno e, muitas vezes, o cerne, onde inoculam um fungo simbionte, que é responsável pelo manchamento; consequentemente, ocorre a desvalorização da madeira (Dorval et al., 2004).

A família Bostrichidae é conhecida por possuir importantes espécies de pragas de grãos e sementes, e de broca de madeira armazenada (Pereira et al., 1997). Estas espécies são conhecidas como "besouros pulverizadores da madeira", ou seja, ao abrirem galerias, transformam a madeira em pó, depreciando o valor de produtos, como peças estruturais e lâminas (Peres Filho et al., 2006).

A presença de espécies de coleobrocas está se tornando relativamente comum em plantações florestais (Silva Paz et al., 2008); dessa forma, estudos que avaliam a ocorrência desses organismos em reflorestamentos se fazem oportunos face às potencialidades de promoção de danos nas espécies florestais e na madeira delas extraída (Flechtmann \& Gaspareto, 1997; Abreu et al., 2002; Zanuncio et al., 2005). Assim, de acordo com Silva Paz et al. (2008), o monitoramento de insetos-pragas no setor florestal é de fundamental importância para que se obtenham informações que sejam utilizadas para a implantação de programas de manejo integrado de pragas, diminuindo, assim, custos de produção por meio da minimização de possíveis danos.

No Brasil, muitos estudos com coleobrocas são efetuados por meio de levantamentos populacionais que, por vezes, são correlacionados a dados climáticos e de vegetação, com ou sem a aplicação de índices faunísticos, utilizando-se armadilhas de impacto; estas podem ser do tipo luminosa ou com atrativo, sendo o atrativo mais comum o álcool etílico (Carvalho et al., 1996; Dall'Oglio \& Peres Filho, 1997). Silveira Neto et al. (1976) afirmaram que o levantamento de insetos é de fundamental importância para estudos ecológicos, pois é praticamente impossível contar todos os insetos de um habitat, sendo tais estudos realizados mediante estimativas de população por meio de amostras.

Em razão da necessidade de informações sobre as espécies de coleópteros que ocorrem associadas às áreas reflorestadas, este trabalho teve como objetivo contribuir para o conhecimento da dinâmica populacional de espécies das subfamílias Scolytinae, Platypodinae e Bostrichinae em um talhão de Eucalyptus camaldulensis, nos períodos de estiagem e de chuva, no município de Cuiabá, Estado de Mato Grosso.

\section{MATERIAL E MÉTODOS}

O estudo foi realizado no período de abril de 2008 a março de 2009, na fazenda Mutuca, da Sadia Frigobrás Indústria e Comércio S.A., localizada no município de Cuiabá, MT. Sua posição geográfica é 
de latitude Sul $15^{\circ} 18^{\prime} 29^{\prime \prime}$ e $55^{\circ} 58^{\prime} 31^{\prime \prime}$ de longitude Oeste, em uma altitude de $800 \mathrm{~m}$, sendo o clima do tipo Aw Köppen. A temperatura média anual é de $26^{\circ} \mathrm{C}$ e a precipitação pluviométrica média anual é de $1400 \mathrm{~mm}$. O solo da região é do tipo areia quartzosa álica, pouco resistente ao intemperismo e com baixa fertilidade. A composição da vegetação natural da região é do tipo cerrado ralo, com campos, matas ripárias e cerradões.

$\mathrm{O}$ experimento foi instalado em um talhão de E. camaldulensis de 22,5 ha com 3,5 anos de idade. Foram utilizadas seis armadilhas etanólicas, modelo escolitídeo-Curitiba modificado pela adoção de um porta-isca constituído de um cano flexível. As armadilhas foram dispostas em duas linhas contendo três armadilhas por linha, guardando entre si uma distância de $30 \mathrm{~m}$ entre armadilhas e nas linhas, e de $50 \mathrm{~m}$ das margens externas dos talhões, para evitar o efeito de borda (Murcia, 1995); nestas, as armadilhas foram instaladas a $1,5 \mathrm{~m}$ da superfície do solo em relação à borda do funil coletor. A cada coleta, o atrativo e o conservante eram renovados, sendo etanol combustível utilizado como atrativo no porta-isca e álcool $70 \%$ como conservante no frasco coletor.

As coletas ocorreram quinzenalmente, porém foram transformadas em mensais para efeito de análises, tais como flutuação populacional e comparação entre os períodos de chuva e estiagem. Os espécimens coletados foram acondicionados em recipientes apropriados, identificados com o número da armadilha e a data de coleta, sendo transportados para o Laboratório de Proteção Florestal da Universidade Federal de Mato Grosso, onde os exemplares foram triados, secos em estufa a $60{ }^{\circ} \mathrm{C}$ por 72 horas, codificados e armazenados.

Os espécimens coletados foram identificados taxonomicamente por comparação com o material entomológico depositado na coleção entomológica do Laboratório de Proteção Florestal, da Faculdade de Engenharia Florestal, da Universidade Federal de Mato Grosso. Também foram enviados exemplares da subfamília Scolytinae para o Laboratório de Proteção Florestal da Universidade Federal do Paraná, para a identificação taxonômica.

A análise quantitativa foi feita mediante contagem direta dos indivíduos. Os índices de diversidade para os períodos de estiagem e de chuva foram calculados utilizando-se o índice de diversidade de Hill, sendo o índice de equitatividade obtido por meio da razão entre $\mathrm{N} 2$ e N1 (número de espécies muito abundantes e número de espécies abundantes, respectivamente), conhecida como Razão de Hill modificada (Hill, 1973). Foram calculados os índices faunísticos de constância, dominância, frequência e abundância para os períodos de estiagem, de chuva e anual, por meio do software ANAFAU (Moraes et al., 2003).

Foram realizados estudos de flutuação populacional com os táxons identificados em nível de espécie e que ocorreram na análise faunística anual como dominantes, muito abundantes, muito frequentes e constantes. Nas análises dos resultados, utilizou-seumdelineamentointeiramentecasualizado em esquema fatorial (Espécies $\times$ Períodos) com seis repetições (armadilhas). Os dados foram submetidos à análise de variância (ANOVA) e as médias foram comparadas entre si pelo teste Scott-Knott no nível de $5 \%$ de probabilidade, utilizando-se o software SAEG (Ribeiro Júnior, 2001). Para efeito de análise estatística, os dados foram transformados pela formula $\log (\mathrm{x}+1)$, conforme recomendações de Banzatto \& Kronka (2008).

\section{RESULTADOS E DISCUSSÃO}

\subsection{Análise qualitativa e quantitativa}

$\mathrm{Na}$ análise conjunta dos períodos (estiagem e chuvoso) ocorreram no talhão de E. camaldulensis 26 espécies de coleópteros, sendo 21 da família Curculionidae (19 da subfamília Scolytinae e duas da Platypodinae) e cinco da família Bostrichidae; a subfamília Scolytinae, em ambos os períodos, foi a mais representativa em quantidade de espécies e de indivíduos coletados, com 17 espécies entre 737 indivíduos nos meses de estiagem e 18 espécies entre 382 indivíduos nos meses de chuva (Tabela 1). Esses resultados corroboram os obtidos por Dorval (2002), que observou em plantios de E. camaldulensis, no município de Cuiabá, MT, uma superior diversidade de espécies nos meses do período de chuva e uma superior quantidade de indivíduos coletados no período de estiagem. 
Tabela 1. Número de espécies e de indivíduos coletados nos períodos de estiagem e chuva no talhão de E. camaldulensis, na fazenda Mutuca, Cuiabá, MT, 2008-2009.

Table 1. Number of species and individuals collected in periods of drought and rain in the stand of E. camaldulensis in the farm Mutuca, Cuiabá, MT, 2008-2009.

\begin{tabular}{|c|c|c|c|c|c|c|c|c|}
\hline \multirow{3}{*}{ Subfamília } & \multicolumn{8}{|c|}{ Período } \\
\hline & \multicolumn{4}{|c|}{ Seco } & \multicolumn{4}{|c|}{ Chuvoso } \\
\hline & $\mathbf{E}$ & $\%$ & I & $\%$ & $\mathbf{E}$ & $\%$ & I & $\%$ \\
\hline Bostrichinae & 5 & 20,83 & 267 & 25,55 & 5 & 20 & 57 & 12,58 \\
\hline Platypodinae & 2 & 8,33 & 41 & 3,92 & 2 & 8 & 14 & 3,09 \\
\hline Scolytinae & 17 & 70,83 & 737 & 70,53 & 18 & 72 & 382 & 84,33 \\
\hline Total & 24 & 100 & 1.045 & 100 & 25 & 100 & 453 & 100 \\
\hline
\end{tabular}

E: espécies. I: indivíduos.

Das 26 espécies encontradas, 23 ocorreram em ambos os períodos. As espécies Tricolus sp. e Coccotripes sp. ocorreram restritas aos períodos de estiagem e chuva, respectivamente. As espécies em que se registraram maiores quantidades de indivíduos no período anual foram Xyleborus affinis e Xyleborus ferrugineus, com 283 e 226 espécimes, respectivamente (Tabela 2). Em estudos realizados por Morales et al. (2000), em um plantio de Eucalyptus grandis (Hill, 1973), constatou-se que essas espécies ( $X$. affinis e $X$. ferrugineus) também foram as mais abundantes, representando 96,27\% de toda a amostra coletada, enquanto que as demais apenas representaram 3,73\%.

Segundo Dorval et al. (2007), o gênero Xyleborus se destaca por apresentar maior número de espécies com potencial de danos, mesmo que apresente espécies que possam ser consideradas benéficas, pois auxiliam na desrama natural de pequenos ramos, contribuindo na degradação de resíduos de madeira dentro de áreas reflorestadas. Esse gênero conta também com inúmeras espécies que causam grande impacto econômico, danificando grandes volumes de madeiras recém-cortadas ou armazenadas aguardando beneficiamento (Wood, 1982; PedrosaMacedo, 1984).

\subsection{Indices de diversidade}

No período de estiagem, houve maior numero de espécies abundantes, apresentando uma maior diversidade em relação ao período de chuva, pois $\mathrm{N} 1$ e N2 apresentaram maiores valores do que no período de chuva. Os índices de equitatividade foram de 0,769 e 0,518 para o período de estiagem e chuvoso, respectivamente (Tabela 3 ), sendo que, na estiagem, obteve-se maior diversidade com maior homogeneidade na distribuição das populações, indicando uma baixa dominância de alguns grupos de insetos onde os recursos ambientais disponíveis são compartilhados de forma igualitária por uma maior quantidade de espécies. Um estudo realizado por Dorval (2002) relatou que há maior diversidade de espécies de coleópteros no período de estiagem em plantios de E. camaldulensis, no município de Cuiabá, MT.

Apesar de o período de chuva ter apresentado maior numero de espécies, o mesmo apresentou o índice de diversidade e equitatibilidade menor, demonstrando que nessa época ocorre uma distribuição irregular dos recursos ambientais disponíveis para os indivíduos, a qual beneficia alguns grupos de insetos que estão adaptados a esse período, fazendo com que o ambiente apresente diversidade e equitatibilidade baixas, nessa época do ano. Esses resultados confirmam o dinamismo temporal das populações em função das possíveis influências intraespecíficas, interespecíficas e dos fatores abióticos.

Essas diferenças confirmam que as populações de coleobrocas distribuem-se espacialmente no ambiente de acordo com seu período de reprodução, quando realizam voos de acasalamento e colonização de novos hospedeiros. De acordo com Chey et al. (1997), as ocorrências de oscilações nas diversidades e na equitatividade entre populações de insetos em ambientes reflorestados podem ser controladas por meio da utilização de métodos de manejo florestal adequado, o que possibilitaria a manutenção de um ambiente ecologicamente mais equilibrado e menos susceptível à ocorrência de surtos populacionais de espécies de pragas. 
Tabela 2. Família, subfamília, espécie e número de indivíduos coletados nos períodos de estiagem, de chuva e anual em um talhão de E. camaldulensis. Fazenda Mutuca, Cuiabá, MT, 2008-2009.

Table 2. Family, subfamily, species and number of individuals collected in the periods of drought, rain and annual in a stand of E. camaldulensis. Farm Mutuca, Cuiabá, MT, 2008-2009.

\begin{tabular}{|c|c|c|c|c|c|c|}
\hline \multirow{3}{*}{ FAMÍLIA/subfamília/espécie } & \multicolumn{6}{|c|}{ Período } \\
\hline & \multicolumn{2}{|c|}{ Seco } & \multicolumn{2}{|c|}{ Chuvoso } & \multicolumn{2}{|c|}{ Anual } \\
\hline & $\bar{I}$ & $\%$ & $\mathbf{I}$ & $\%$ & Total & $\%$ \\
\hline \multicolumn{7}{|l|}{ BOSTRICHIDAE } \\
\hline \multicolumn{7}{|l|}{ Bostrichinae } \\
\hline Bostrychopsis laminifer (Lesne, 1895) & 1 & 0,10 & 2 & 0,44 & 3 & 0,20 \\
\hline Bostrychopsis uncinata (German, 1824) & 96 & 9,19 & 30 & 6,62 & 126 & 8,41 \\
\hline Micrapate sp. & 134 & 12,82 & 6 & 1,32 & 140 & 9,35 \\
\hline Xyloperthela picea (Oliver, 1790) & 24 & 2,30 & 4 & 0,88 & 28 & 1,87 \\
\hline Xyloprista sp. & 12 & 1,15 & 15 & 3,31 & 27 & 1,80 \\
\hline \multicolumn{7}{|l|}{ CURCULIONIDAE } \\
\hline \multicolumn{7}{|l|}{ Platypodinae } \\
\hline Platypus linearis Chapuis, 1865 & 40 & 3,83 & 13 & 2,87 & 53 & 3,54 \\
\hline Platypus sp. & 1 & 0,10 & 1 & 0,22 & 2 & 0,13 \\
\hline \multicolumn{7}{|l|}{ Scolytinae } \\
\hline Coccotrypes palmarum Eggers, 1933 & 11 & 1,05 & 5 & 1,10 & 16 & 1,07 \\
\hline Corthylus sp. & - & - & 1 & 0,22 & 1 & 0,07 \\
\hline Cryptocarenus diadematus Eggers, 1937 & 43 & 4,11 & 10 & 2,21 & 53 & 3,54 \\
\hline Cryptocarenus heveae (Hagedorni, 1912) & 25 & 2,39 & 17 & 3,75 & 42 & 2,80 \\
\hline Cryptocarenus seriatus Eggers, 1933 & 75 & 7,18 & 16 & 3,53 & 91 & 6,07 \\
\hline Cryptocarenus sp. & 2 & 0,19 & 7 & 1,55 & 9 & 0,60 \\
\hline Hypothenemus bolivianus Eggers, 1931 & 15 & 1,44 & 1 & 0,22 & 16 & 1,07 \\
\hline Hypothenemus eruditus Westwood, 1836 & 107 & 10,24 & 20 & 4,42 & 127 & 8,48 \\
\hline Hypothenemus obscurus (Fabricius, 1801) & 20 & 1,91 & 14 & 3,09 & 34 & 2,27 \\
\hline Microcorthylus sp. & - & - & 1 & 0,22 & 1 & 0,07 \\
\hline Premnobius cavipennis Eichhoff, 1867 & 58 & 5,55 & 24 & 5,30 & 82 & 5,47 \\
\hline Sampsonius dampfi Schedl, 1940 & 3 & 0,29 & 2 & 0,44 & 5 & 0,33 \\
\hline Xyleborus affinis Eichhoff, 1867 & 200 & 19,14 & 83 & 18,32 & 283 & 18,89 \\
\hline Xyleborus compactus Eichhoff, 1875 & 5 & 0,48 & 4 & 0,88 & 9 & 0,60 \\
\hline Xyleborus ferrugineus (Fabricius, 1801) & 63 & 6,03 & 163 & 35,95 & 226 & 15,09 \\
\hline Xyleborus retusus Eichhoff, 1868 & 44 & 4,21 & 3 & 0,66 & 47 & 3,14 \\
\hline Xyleborus spinosulus Schedl, 1934 & 3 & 0,29 & 4 & 0,88 & 7 & 0,47 \\
\hline Xyleborus spinulosus Schedl, 1934 & 62 & 5,93 & 7 & 1,55 & 69 & 4,61 \\
\hline Tricolus sp. & 1 & 0,10 & - & - & 1 & 0,07 \\
\hline Total & 1.045 & 100 & 453 & 100 & 1.498 & 100 \\
\hline
\end{tabular}

Tabela 3. Índice de diversidade e equitatibilidade de coleópteros coletados em talhão de E. camaldulensis, nos períodos de estiagem e de chuva. Fazenda Mutuca, Cuiabá, MT, 2008-2009.

Table 3. Diversity index and equitability of coleoptera collected in a stand of E. camaldulensis in the periods of drought and rain. Farm Mutuca, Cuiabá, MT, 2008-2009.

\begin{tabular}{ccc|} 
Índice de & \multicolumn{2}{c|}{ Períodos } \\
\cline { 2 - 3 } diversidade & Seco & Chuvoso \\
\cline { 2 - 3 } N0 & 24 & 25 \\
N1 & 13,618 & 9,932 \\
N2 & 10,705 & 5,633 \\
H' & 2,6114 & 2,295 \\
E & 0,769 & 0,518 \\
\hline
\end{tabular}

N0 = número total de espécies; N1 = número de espécies abundantes; N2 = número de espécies muito abundantes; H’ = Shannon Wiener; E = equitatibilidade (Razão modificada de Hill's). 


\subsection{Estudo faunístico}

Ocorreram oito espécies dominantes, cinco muito abundantes, seis muito frequentes e 17 constantes. No período de estiagem, nove foram dominantes, cinco muito abundantes e muito frequentes, e 18 constantes, enquanto que nos meses de chuva, cinco ocorreram como dominantes, duas como muito abundantes e muito frequentes e 15 constantes. Apenas X. affinis ocorreu como dominante, muito abundante, muito frequente e constante, independentemente do período analisado. As espécies B. uncinata, Micrapate sp., C. seriatus e $H$. eruditus ocorreram como dominantes, muito abundantes, muito frequentes e constantes somente no período de estiagem, enquanto $X$. ferrugineus ocorreu como dominante, muito abundante, muito frequente e constante somente no período de chuva (Tabela 4).

Dorval (2002) realizou estudos faunísticos com espécies de coleópteros em um talhão de E. camaldulensis em Cuiabá, MT, no qual as espécies C. heveae, C. seriatus, H. obscurus, H. eruditus e C. diadematus ocorreram como dominantes, constantes, muito abundantes e com altas frequências de indivíduos coletados, independentemente do período de coletas. Müller \& Andreiv (2004)

Tabela 4. Índices faunísticos das espécies coletadas em talhão de E. camaldulensis nos períodos de estiagem, de chuva e anual. Fazenda Mutuca, Cuiabá, MT, 2008-2009.

Table 4. Faunistic index the species collected in a stand of E. camaldulensis in the periods of drought, rain and annual. Farm Mutuca, Cuiabá, MT, 2008-2009.

\begin{tabular}{|c|c|c|c|c|c|c|c|c|c|c|c|c|}
\hline \multirow{3}{*}{ Espécies } & \multicolumn{12}{|c|}{ Período } \\
\hline & \multicolumn{4}{|c|}{ Seca } & \multicolumn{4}{|c|}{ Chuva } & \multicolumn{4}{|c|}{ Anual } \\
\hline & D & A & $\mathbf{F}$ & $\mathrm{C}$ & D & $\mathbf{A}$ & $\mathbf{F}$ & $\mathrm{C}$ & D & A & $\mathbf{F}$ & $\mathrm{C}$ \\
\hline Bostrychopsis laminifer & nd & $\mathrm{r}$ & $\mathrm{pf}$ & $\mathrm{z}$ & nd & $\mathrm{d}$ & $\mathrm{pf}$ & $\mathrm{y}$ & nd & $\mathrm{r}$ & $\mathrm{pf}$ & $\mathrm{y}$ \\
\hline Bostrychopsis uncinata & $\mathrm{d}$ & $\mathrm{ma}$ & $\mathrm{mf}$ & $\mathrm{w}$ & $\mathrm{d}$ & c & $\mathrm{F}$ & $\mathrm{w}$ & $\mathrm{d}$ & $\mathrm{ma}$ & $\mathrm{mf}$ & w \\
\hline Micrapate sp. & $\mathrm{d}$ & $\mathrm{ma}$ & $\mathrm{mf}$ & $\mathrm{w}$ & nd & c & $\mathrm{F}$ & $\mathrm{w}$ & d & $\mathrm{ma}$ & $\mathrm{mf}$ & $\mathrm{w}$ \\
\hline Xyloperthela picea & nd & c & $\mathrm{f}$ & $\mathrm{w}$ & nd & d & $\mathrm{pf}$ & $\mathrm{y}$ & nd & $\mathrm{d}$ & $\mathrm{pf}$ & w \\
\hline Xyloprista sp. & nd & $\mathrm{r}$ & $\mathrm{pf}$ & $\mathrm{w}$ & nd & c & $\mathrm{F}$ & $\mathrm{w}$ & nd & d & $\mathrm{pf}$ & $\mathrm{w}$ \\
\hline Platypus linearis & nd & c & $\mathrm{f}$ & $\mathrm{w}$ & nd & c & $\mathrm{F}$ & $\mathrm{w}$ & nd & c & $\mathrm{f}$ & w \\
\hline Platypus sp. & nd & $\mathrm{r}$ & $\mathrm{pf}$ & $\mathrm{z}$ & nd & $\mathrm{d}$ & $\mathrm{pf}$ & $\mathrm{z}$ & nd & $\mathrm{r}$ & $\mathrm{pf}$ & $\mathrm{z}$ \\
\hline Coccotrypes palmarum & nd & $\mathrm{r}$ & $\mathrm{pf}$ & $\mathrm{w}$ & nd & c & $\mathrm{F}$ & $\mathrm{w}$ & nd & $\mathrm{r}$ & $\mathrm{pf}$ & $\mathrm{w}$ \\
\hline Corthylus sp. & - & - & - & - & nd & $\mathrm{d}$ & $\mathrm{pf}$ & $\mathrm{z}$ & nd & $\mathrm{r}$ & $\mathrm{pf}$ & $\mathrm{z}$ \\
\hline Cryptocarenus diadematus & nd & c & $\mathrm{f}$ & $\mathrm{w}$ & nd & c & $\mathrm{F}$ & $\mathrm{w}$ & nd & c & $\mathrm{f}$ & $\mathrm{w}$ \\
\hline Cryptocarenus heveae & nd & c & $\mathrm{f}$ & $\mathrm{w}$ & nd & c & $\mathrm{F}$ & $\mathrm{w}$ & nd & c & $\mathrm{f}$ & w \\
\hline Cryptocarenus seriatus & $\mathrm{d}$ & $\mathrm{ma}$ & $\mathrm{mf}$ & $\mathrm{w}$ & nd & c & $\mathrm{F}$ & $\mathrm{w}$ & d & $\mathrm{a}$ & $\mathrm{mf}$ & $\mathrm{w}$ \\
\hline Cryptocarenus sp. & nd & $\mathrm{r}$ & $\mathrm{pf}$ & $\mathrm{z}$ & nd & $c$ & $\mathrm{~F}$ & $\mathrm{z}$ & nd & $\mathrm{r}$ & $\mathrm{pf}$ & $\mathrm{z}$ \\
\hline Hypothenemus bolivianus & nd & $\mathrm{r}$ & $\mathrm{pf}$ & $\mathrm{w}$ & nd & d & $\mathrm{pf}$ & $\mathrm{z}$ & nd & $\mathrm{r}$ & $\mathrm{pf}$ & $\mathrm{w}$ \\
\hline Hypothenemus eruditus & $\mathrm{d}$ & $\mathrm{ma}$ & $\mathrm{mf}$ & $\mathrm{w}$ & $\mathrm{d}$ & c & $\mathrm{F}$ & $\mathrm{w}$ & d & $\mathrm{ma}$ & $\mathrm{mf}$ & $\mathrm{w}$ \\
\hline Hypothenemus obscurus & nd & $\mathrm{d}$ & $\mathrm{pf}$ & $\mathrm{w}$ & nd & c & $\mathrm{F}$ & $\mathrm{w}$ & nd & c & $\mathrm{f}$ & w \\
\hline Microcorthylus sp. & - & - & - & - & nd & $\mathrm{d}$ & $\mathrm{pf}$ & $\mathrm{z}$ & nd & $\mathrm{r}$ & $\mathrm{pf}$ & $\mathrm{z}$ \\
\hline Premnobius cavipennis & $\mathrm{d}$ & c & $\mathrm{f}$ & $\mathrm{w}$ & $\mathrm{d}$ & c & $\mathrm{F}$ & $\mathrm{w}$ & d & c & $\mathrm{f}$ & $\mathrm{w}$ \\
\hline Sampsonius dampfi & nd & $\mathrm{r}$ & $\mathrm{pf}$ & $\mathrm{w}$ & nd & d & $\mathrm{pf}$ & $\mathrm{y}$ & nd & $\mathrm{r}$ & $\mathrm{pf}$ & $\mathrm{y}$ \\
\hline Xyleborus affinis & $\mathrm{d}$ & $\mathrm{ma}$ & $\mathrm{mf}$ & $\mathrm{w}$ & $\mathrm{d}$ & $\mathrm{ma}$ & $\mathrm{mf}$ & $\mathrm{w}$ & d & $\mathrm{ma}$ & $\mathrm{mf}$ & w \\
\hline Xyleborus compactus & nd & $\mathrm{r}$ & $\mathrm{pf}$ & $\mathrm{y}$ & nd & $\mathrm{d}$ & $\mathrm{pf}$ & $\mathrm{y}$ & nd & $\mathrm{r}$ & $\mathrm{pf}$ & $\mathrm{y}$ \\
\hline Xyleborus ferrugineus & $\mathrm{d}$ & c & $\mathrm{f}$ & $\mathrm{w}$ & $\mathrm{d}$ & $\mathrm{ma}$ & $\mathrm{mf}$ & $\mathrm{w}$ & $\mathrm{d}$ & $\mathrm{ma}$ & $\mathrm{mf}$ & w \\
\hline Xyleborus retusus & $\mathrm{d}$ & c & $\mathrm{f}$ & $\mathrm{w}$ & nd & d & $\mathrm{pf}$ & $\mathrm{w}$ & nd & c & $\mathrm{f}$ & $\mathrm{w}$ \\
\hline Xyleborus spinosulus & nd & $\mathrm{r}$ & $\mathrm{pf}$ & $\mathrm{y}$ & nd & d & $\mathrm{pf}$ & $\mathrm{z}$ & nd & $\mathrm{r}$ & $\mathrm{pf}$ & $\mathrm{y}$ \\
\hline Xyleborus spinulosus & $\mathrm{d}$ & c & $\mathrm{f}$ & $\mathrm{w}$ & nd & c & $\mathrm{F}$ & $\mathrm{w}$ & $\mathrm{d}$ & c & $\mathrm{f}$ & $\mathrm{w}$ \\
\hline Tricolus sp. & nd & $\mathrm{r}$ & $\mathrm{pf}$ & $\mathrm{z}$ & - & - & - & - & nd & $\mathrm{r}$ & $\mathrm{pf}$ & $\mathrm{z}$ \\
\hline
\end{tabular}

D: Dominância Sakagami \& Laroca (1967) - (sd) super dominante; (d) dominante; (nd) não dominante. A: Abundância - (sa) super abundante; (ma) muito abundante; (a) abundante; (c) comum; (d) dispersa; (r) rara. F: Frequência - (sf) super frequente; (mf) muito frequente; (f) frequente; (pf) pouco frequente. C: Constância - (w) constante; (y) acessória; (z) acidental. 
relataram em um plantio de E. grandis no município de Blumenau, no Estado de Santa Catarina, as espécies $H$. eruditus, $X$. affinis e $X$. ferrugineus como dominantes e abundantes no período total de coleta. Estudos realizados por Peres Filho et al. (2005), em plantios de Eucalyptus spp, no município de Rondonópolis, no Estado de Mato Grosso, constataram a ocorrência de oito espécies das famílias Bostrichidae e Curculionidae, em que $X$. picea (Bostrichinae), P. cavipennis e X. spinosulus (Scolytinae) ocorreram como constantes e dominantes.

\subsection{Flutuação populacional}

A maioria das espécies ocorreu com picos populacionais no período de estiagem, talvez em função de uma maior abundância de material lenhoso disponível e de hospedeiros estressados por causa do longo período de estiagem - que é característico dessa região nesses meses do ano - e que acabam ficando susceptíveis à colonização por diferentes espécies de coleobrocas.

$\mathrm{Na}$ análise faunística, as espécies descritas como dominante, muito abundante, muito frequente e constante no período anual foram as espécies B. uncinata, $H$. eruditus, $X$. affinis e $X$. ferrugineus, sendo que $B$. uncinata e $H$. eruditus apresentaram picos populacionais em outubro e em agosto, respectivamente (Figura 1). Flechtmann et al. (1996) encontraram resultados semelhantes em plantios de Pinus spp. no município de Agudos, SP, onde essas espécies ocorreram com picos populacionais em outubro, período de seca na região.

Dall'Oglio e Peres Filho (1997), estudando flutuações populacionais de coleobrocas em plantio clonal de seringueira, no município de Itiquira, MT, observaram maiores picos populacionais das espécies $B$. uncinata e de $H$. eruditus nos meses de estiagem (maio a setembro). A espécie $X$. affinis acorreu com picos populacionais em julho e outubro, enquanto $X$. ferrugineus diferenciou-se das demais, ocorrendo com um pico populacional em janeiro, mês de chuvas nesta região (Figura 2).

Resultado semelhante foi observado por Diaz (1996) em talhões de E. citriodora, onde $X$. ferrugineus ocorreu com picos em janeiro, março e maio, meses estes de altas precipitações pluviais

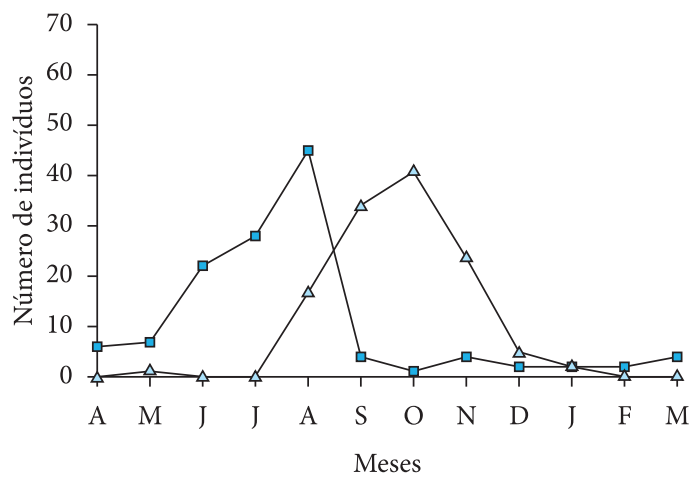

Figura 1. Flutuação populacional de Bostrichopsis uncinata (Bostrichinae) e Hypothenemus eruditus (Scolytinae) em talhão de E. camaldulensis. Fazenda Mutuca, Cuiabá, MT, 20082009.

Figure 1. Population fluctuation of Bostrichopsis uncinata (Bostrichinae) and Hypothenemus eruditus (Scolytinae) in a stand of E. camaldulensis. Farm Mutuca, Cuiabá, MT, 2008-2009.

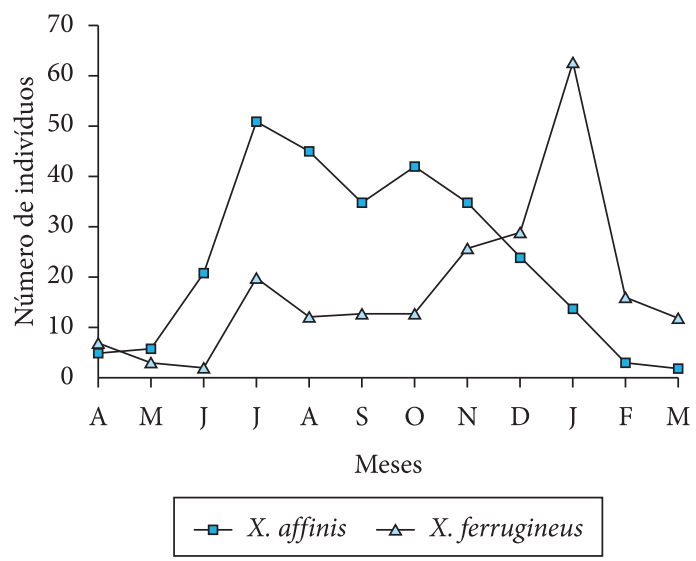

Figura 2. Flutuação populacional de Xyleborus affinis e Xyleborus ferrugineus (Scolytinae) em talhão de E. camaldulensis. Fazenda Mutuca, Cuiabá, MT, 20082009.

Figure 2. Population fluctuation of Xyleborus affinis and Xyleborus ferrugineus (Scolytinae) in a stand of E. camaldulensis. Farm Mutuca, Cuiabá, MT, 20082009.

na área de estudo. Dall'Oglio \& Peres Filho (1997) obtiveram resultados diferentes em plantio clonal de seringueira, no município de Itiquira, MT, onde $X$. ferrugineus ocorreu com picos populacionais nos meses de estiagem dessa região, indicando que as ocorrências e as densidades populacionais dessa 
espécie podem ser influenciadas pela diversidade de espécies florestais hospedeiras.

\subsection{Análise de variância e teste de médias}

Foram observadas diferenças estatísticas significativas entre os fatores estudados (Espécie $\times$ Período). Ocorreu um agrupamento de dez espécies, formando um grupo em que, mesmo apresentando quantidades médias altas e baixas de indivíduos coletados, essas espécies não se diferenciaram significativamente entre os períodos estudados, demonstrando que os períodos não afetam a ocorrência desse grupo de insetos (Tabela 5).
No período de estiagem, as espécies Micrapate sp. e $X$. affinis apresentaram maiores quantidades de indivíduos, não se diferenciando significativamente entre si. No período chuvoso, $X$. ferrugineus foi a espécie mais importante, pois apresentou maior quantidade de indivíduos e foi significativamente maior que as demais espécies nesse período. Foi possível constatar os períodos interferem na ocorrência de alguns insetos, pois foram observados que 13 espécies apresentaram diferenças significativas entre os períodos de estiagem e de chuva.

Dorval \& Peres Filho (2001) encontraram diferenças estatísticas entre os indivíduos de coleópteros coletados em ambiente de Cerrado do

Tabela 5. Número médio de indivíduos coletados no talhão de Eucalyptus camaldulensis, nos períodos de seca e de chuva. Fazenda Mutuca, Cuiabá-MT, 2008-2009.

Table 5. Number media of individuals collected in a stand of Eucalyptus camaldulensis in the periods of drought and rain. Farm Mutuca, Cuiabá, MT, 2008-2009.

\begin{tabular}{|c|c|c|c|c|}
\hline \multirow{3}{*}{$\begin{array}{r}\text { Espécies } \\
\text { Bostrychopsis laminifer }\end{array}$} & \multicolumn{4}{|c|}{ Períodos $^{1}$} \\
\hline & \multicolumn{2}{|c|}{ Seco } & \multicolumn{2}{|c|}{ Chuva } \\
\hline & 0,0502 & $\mathrm{Fa}$ & 0,1003 & $\mathrm{Da}$ \\
\hline Bostrychopsis uncinata & 1,1495 & $\mathrm{Ba}$ & 0,6476 & $\mathrm{Cb}$ \\
\hline Micrapate sp. & 1,3600 & $\mathrm{Aa}$ & 0,2168 & $\mathrm{Db}$ \\
\hline Xyloperthela picea & 0,6693 & $\mathrm{Da}$ & 0,1799 & $\mathrm{Db}$ \\
\hline Xyloprista sp. & 0,4601 & $\mathrm{Ea}$ & 0,5102 & $\mathrm{Ca}$ \\
\hline Platypus linearis & 0,8666 & $\mathrm{Ca}$ & 0,4894 & $\mathrm{Cb}$ \\
\hline Platypus sp. & 0,0502 & $\mathrm{Fa}$ & 0,0502 & $\mathrm{Da}$ \\
\hline Coccotrypes palmarum & 0,3967 & $\mathrm{Ea}$ & 0,2300 & $\mathrm{Da}$ \\
\hline Corthylus sp. & - & - & 0,0502 & $\mathrm{D}$ \\
\hline Cryptocarenus diadematus & 0,8406 & $\mathrm{Ca}$ & 0,3465 & $\mathrm{Cb}$ \\
\hline Cryptocarenus heveae & 0,6681 & $\mathrm{Da}$ & 0,5132 & $\mathrm{Ca}$ \\
\hline Cryptocarenus seriatus & 1,0890 & $\mathrm{Ba}$ & 0,5472 & $\mathrm{Cb}$ \\
\hline Cryptocarenus sp. & 0,1003 & $\mathrm{Fa}$ & 0,2594 & $\mathrm{Da}$ \\
\hline Hypothenemus bolivianus & 0,4666 & $\mathrm{Ea}$ & 0,0502 & $\mathrm{Db}$ \\
\hline Hypothenemus eruditus & 1,2709 & $\mathrm{Ba}$ & 0,5719 & $\mathrm{Cb}$ \\
\hline Hypothenemus obscurus & 0,5604 & $\mathrm{Ea}$ & 0,4686 & $\mathrm{Ca}$ \\
\hline Microcorthylus sp. & - & - & 0,0502 & $\mathrm{D}$ \\
\hline Premnobius cavipennis & 0,8447 & $\mathrm{Ca}$ & 0,5835 & $\mathrm{Cb}$ \\
\hline Sampsonius dampfi & 0,1505 & $\mathrm{Fa}$ & 0,1003 & $\mathrm{Da}$ \\
\hline Xyleborus affinis & 1,5256 & $\mathrm{Aa}$ & 1,1141 & $\mathrm{Bb}$ \\
\hline Xyleborus compactus & 0,2007 & $\mathrm{Fa}$ & 0,1799 & $\mathrm{Da}$ \\
\hline Xyleborus ferrugineus & 1,0262 & $\mathrm{Cb}$ & 1,4026 & $\mathrm{Aa}$ \\
\hline Xyleborus retusus & 0,9015 & $\mathrm{Ca}$ & 0,1003 & $\mathrm{Db}$ \\
\hline Xyleborus spinosulus & 0,1505 & $\mathrm{Fa}$ & 0,1505 & $\mathrm{Da}$ \\
\hline Xyleborus spinulosus & 1,0166 & $\mathrm{Ca}$ & 0,3304 & $\mathrm{Cb}$ \\
\hline Tricolus sp. & 0,0502 & $\mathrm{~F}$ & - & - \\
\hline $\mathrm{F}$ & & & & \\
\hline
\end{tabular}

${ }^{1}$ Dados Transformados $\log (\mathrm{X}+1)$. Médias acompanhadas de mesma letra maiúscula nas colunas e minúscula nas linhas não diferem entre si no nível de 5\% de probabilidade pelo teste Scott-Knott. 
município de Cuiabá, no Estado de Mato Grosso, no qual as espécies H. obscurus, C. heveae, C. diadematus e C. seriatus formaram um grupo de maior quantidade de indivíduos, sendo estatisticamente diferentes das demais espécies. Lunz e Carvalho (2002) estudaram a ocorrência de coleobrocas em seis espécies florestais em Seropédica, Estado do Rio de Janeiro, e observaram uma alta frequência de $X$. affinis e $X$. ferrugineus em amostras de lenho, e de $H$. eruditus em amostras de casca, sendo que, pelo teste de Tukey, Xyleborus intricatus diferenciouse quantitativamente das demais, enquanto $X$. ferrugineus, X. affinis, Theoborus villosulus, P. cavipennis e Ambrosiodmus obliquus não apresentaram diferenças estatísticas significativas entre si.

\section{CONCLUSÕES}

Das 26 espécies coletadas, 19 pertencem à subfamília Scolytinae, a qual foi a mais representativa em ambos os períodos.

O período de estiagem propicia condições para a ocorrência de uma maior diversidade e maior uniformidade na comunidade de coleobrocas presentes em reflorestamento de Eucalyptus camaldulensis, bem como maiores quantidades de indivíduos.

O período de chuva propicia condições para que ocorra uma maior riqueza de espécies, porém com desuniformidade na ocorrência destas, o que proporciona condições para promover um menor índice de diversidade em relação ao período de estiagem.

$\mathrm{Na}$ análise faunística, destacaram-se as espécies Bostrychopsis uncinata, Hypothenemus eruditus, Premnobius cavipennis, Xyleborus affinis e Xyleborus ferrugineus, pois estas foram dominantes em ambos os períodos, bem como no período anual.

\section{AGRADECIMENTOS}

Ao Prof. Dr. Eli Nunes Marques da Universidade Federal do Paraná (UFPR), pela identificação das espécies da subfamília Scolytinae, ao Prof. Dr. Joadil Gonçalves de Abreu da Faculdade de Agronomia e Medicina Veterinária (FAMEV-UFMT), pelas análises estatísticas realizadas, e ao técnico de Laboratório Manoel Lauro da Silva, pelo inestimável auxílio no desenvolvimento deste trabalho.

\section{STATUS DA SUBMISSÃO}

Recebido: 11/07/2011

Aceito: 06/10/2011

Resumo publicado online: 07/10/2011

Artigo completo publicado: 22/12/2011

\section{AUTOR(ES) PARA CORRESPONDÊNCIA}

\section{José Renato Maurício da Rocha}

Programa de Pós-Graduação em Ciências Florestais e Ambientais, Faculdade de Engenharia Florestas - FENF, Universidade Federal de Mato Grosso - UFMT Av. Fernando Corrêa da Costa, 2367, Boa Esperança, CEP 78125-070, Cuiabá, MT, Brasil e-mail: jrm_rocha@hotmail.com

\section{Alberto Dorval \\ Departamento de Engenharia Florestal, Faculdade de Engenharia Florestas - FENF, Universidade Federal de Mato Grosso - UFMT Av. Fernando Corrêa da Costa, 2367, Boa Esperança, CEP 78125-070, Cuiabá, MT, Brasil e-mail: adorval@terra.com.br}

\section{REFERENNCIAS}

Abreu RLS, Sales-Campos C, Hanada RE, Vasconcelos FJ, Freitas JA. Avaliação de danos por insetos em toras estocadas em indústrias madeireiras de Manaus, Amazonas, Brasil. Revista Árvore 2002; 26(6): 789-796.

Banzatto DA, Kronka SN. Experimentação Agrícola. 4rd ed. Jaboticabal: Funep; 2008. 237 p.

Carvalho AG, Rocha MP, Silva CAM, Lunz AM. Variação sazonal de Scolytidae (Coleoptera) numa comunidade de floresta natural de Seropédica, RJ. Floresta e Ambiente 1996; 3(2): 9-14.

Chey VK, Holloway JD, Speight MR. Diversity of moths in forest plantations and natural forests in Sabah. Bulletin of Entomological Research 1997; 87(1): 371-385. http://dx.doi.org/10.1017/S000748530003738X

Dall'oglio OT, Peres Filho O. Levantamento e flutuação de populacional de coleobrocas em plantios homogêneos de seringueira em Itiquira-MT. Scientia Forestalis 1997; 51(2): 49-58. 
Del Quiqui EM, Martins SS, Shimizu JY. Avaliação de espécies e procedências de Eucalyptus spp para o Noroeste do Estado do Paraná. Acta Scientiarum 2001; 23(5): 1173-1177.

Diaz EAB. Análise faunística de Scolytidae, Platypodidae e Bostrichidae (Coleptera) em comunidades florestais no departamento de Guairá, Paraguai [dissertação]. Curitiba: Universidade Federal do Paraná; 1996.

Dorval A. Levantamento populacional de coleópteros com armadilhas etanólicas em plantios de eucaliptos e em uma área com vegetação de cerrado no município de Cuiabá, Estado de Mato Grosso [tese]. Curitiba: Universidade Federal do Paraná; 2002.

Dorval A, Peres Filho O, Marques EN. Levantamento de Scolytidae (Coleoptera) em plantações de Eucalyptus spp. em Cuiabá, estado de Mato Grosso. Ciência Florestal 2004; 14(1): 47-58.

Dorval, A, Peres Filho, O, Marques, E. N, Berti Filho, E, Moura, R. G. Infestação de coleobrocas em madeiras de Eucalyptus spp. em Cuiabá, Estado de Mato Grosso. Revista de Agricultura 2007; 82(2): 134-141.

Dorval A, Peres Filho O. Levantamento e flutuação populacional de coleópteros em vegetação do cerrado da baixada Cuiabana, MT. Ciência Florestal 2001; 11(2): 171-182.

Flechtmann CAH, Gaspareto CL. Scolytidae em pátio de serraria da fábrica Paula Souza (Botucatu/SP) e fazenda Rio Claro (Lençóis Paulista/SP). Scientia Forestalis 1997; 51(2): 61-75.

Flechtmann CAH, Teixeira EP, Gaspareto CL. Bostrichidae (Coleoptera) capturados em armadilhas iscadas com etanol em pinheiros tropicais na região de Agudos-SP. Revista Instituto Florestal 1996; 8(1): 17-44.

Hill MO. Diversity and evenness: a unifying notation and its consequences. Ecology 1973; 54(2): 427-432. http://dx.doi.org/10.2307/1934352

Lunz AM, Carvalho AG. Degradação da madeira de seis essências arbóreas dispostas perpendicularmente ao solo, causada por Scolytidae (Coleoptera). Neotropical Entomology 2002; 31(3): 351-357. http://dx.doi. org/10.1590/S1519-566X2002000300002

Moraes MLT, Higa AR, Cavenage A, Kano NK. Avaliação da densidade básica da madeira e de sua relação com os caracteres de crescimento, em uma população base de Eucalyptus camaldulensis Dehn. In: Anais da Conferência IUFRO sobre silvicultura e melhoramento de eucaliptos; 1997; Salvador. Colombo: EMBRAPA/CNPF; 1997. v. 3, n. 1, p.43-47.

Moraes RCB, Haddad ML, Silveira Neto S, Reyes AEL. Software para análise faunística. In: Anais do $8 \mathrm{rd}$ Simpósio de Controle Biológico; 2003; São Paulo. Piracicaba: ESALQ; 2003. p. 95

Morales EN, Zanuncio JC, Pratissoli D, Fabres AS. lutuação populacional de Scolytidae (Coleoptera) em áreas reflorestadas com Eucalyptus grandis (Myrtaceae) em Minas Gerais, Brasil. Revista de Biología Tropical 2000; 48(1): 2000.

Müller JA, Andreiv J. Caracterização da família Scolytidae (Insecta: Coleoptera) em três ambientes florestais. Cerne 2004; 10(1): 39-45.

Murcia C. Edge effects in fragmented forests: implications for conservation. Tree Reviews. 1995; 10(2): 58-62.

Oliveira HG, Zanuncio TV, Zanuncio JC, Santos GP. Coleópteros associados à eucaliptocultura na região de nova era, Minas Gerais, Brasil. Floresta e Ambiente 2001; 8(1): 52-60.

Pedrosa-Macedo JH. Risco da não utilização de resíduos florestais. In: Anais do 5rd Curso de atualização sobre sistemas de exploração e transporte florestal; 1984; Curitiba. Curitiba: FUPEF; 1984. p. 40-49.

Pereira PRVS, Furiatti RS, Lazzari FA, Pinto Junior AR. Avaliação de Inseticidas no Controle de Sitophilus oryzae (L.) (Coleoptera: Curculionidae), e Rhyzopertha dominica (Fab.) (Coleoptera: Bostrichidae) em Milho Armazenado. Anais da Sociedade Entomológica do Brasil 1997; 26(3): 411-416. http://dx.doi.org/10.1590/ S0301-80591997000300001

Peres Filho O, Dorval A, Siqueira SA, Berti Filho E. Levantamento de coleópteros em plantios de Eucalyptus spp. em Rondonópolis, estado de Mato Grosso. Revista da Agricultura 2005; 80(2): 213-227.

Peres Filho O, Dorval A, Berti Filho E. A Entomofauna Associada à Teca, Tectona grandis L.f. no Estado de Mato Grosso. Piracicaba: IPEF; 2006. 58 p.

Ribeiro Júnior JI. Análises estatísticas no SAEG. Viçosa: UFV; 2001. 301 p.

Sakagami SF, Laroca, S. Observations on the bionomics of some neotropical Xylocopini bees, with some comparative biofaunistic notes (Hymenoptera, Anthophoridae). Journal of the Faculty of Science 1967; 18: 57-127.

Silva Paz JK, Silva PRR, Pádua LEM, IdeS, Carvalho EMS, Feitosa SS. Monitoramento de coleobrocas associadas à mangueira no município José de Freitas, estado do Piauí. Revista Brasileira de Fruticultura 2008; 30(2): 348-355.

Silveira Neto S, Nakano O, Barbin D. Manual de ecologia dos insetos. São Paulo: Agronômica Ceres; 1976. 419 p.

Wood SL. The bark and ambrosia beetles of North and Central America (Coleoptera: Scolytidae), a taxonomic monograph. Great Basin Naturalist Memorandum 1982; 6: 1-1359.

Zanuncio JC, Sossai MF, Flechtmann CAH, Zanuncio TV, Guimarães EM, Espindula MC. Plants of an Eucalyptus clone damage by Scolytidae and Platypodidae (Coleoptera). Pesquisa Agropecuária Brasileira 2005; 40(2): 513-515. 